\title{
microRNA-29a-3p, Up-Regulated in Human Gastric Cells and Tissues with H.Pylori Infection, Promotes the Migration of GES-1 Cells via A20-Mediated EMT Pathway
}

\author{
Fengying Sun ${ }^{\mathrm{a}, \mathrm{b}}$ Ying $\mathrm{Ni}^{\mathrm{a}}$ Hong Zhu ${ }^{\mathrm{a}}$ Jian Fang ${ }^{\mathrm{c}}$ Hua Wang ${ }^{\mathrm{a}}$ Jinxuan Xia ${ }^{\mathrm{a}}$ \\ Fangfang Ding $^{a}$ HongxingShen $^{a}$ Shihe Shao ${ }^{a}$ \\ aSchool of Medicine, Jiangsu University, Zhenjiang, Jiangsu, bDepartment of Clinical Laboratory, The \\ Second People's Hospital of Wuhu, Anhui, 'Department of Transfusion, The first affiliated hospital of \\ Anhui medical university, Hefei, China
}

\section{Key Words}

Helicobacter pylori • miR-29a-3p • A20 • Migration • EMT

\begin{abstract}
Background/Aims: Helicobacter pylori (H. pylori) infection is closely related to human gastric mucosa-associated diseases. Several recent studies on miRNAs have expanded our insights on H.pylori pathogenesis. This study aimed to investigate the biological roles and underlying molecular mechanisms of miR-29a-3p in human gastric cells and tissues with H.pylori infection. Methods: miR-29a-3p expression was quantified by quantitative RT-PCR (qRT$P C R)$. A miR-29a-3p target gene was validated by bioinformatics analysis, western blotting and dual luciferase reporter gene assays. Western blotting and immunohistochemistry (IHC) assay were performed to detect the protein expression. Transwell assay was used to determine the cell migration ability. Results: MiR-29a-3p was up-regulated in H.pylori-positive gastric mucosa tissues and H.pylori-infected gastric cells. The up-regulation of miR-29a-3p was dose-dependent in BGC-823 and GES-1 cells infected with H.pylori. Using gain- and loss-offunction experiments in vitro, we demonstrated that miR-29a-3p promoted the migration of gastric epithelial cells. We further characterized A20 as a direct target of miR-29a-3p. The expression of A20 was decreased in H.pylori-positive gastric mucosa tissues compared with H.pylori-negative gastric mucosa tissues. A20 downregulation was time- and dose-dependent in GES-1 and BGC-823 cells infected with H.pylori. In GES-1 and BGC-823 cells infected with H.pylori, the miR-29a-3p mimic significantly blocked A20 expression, which suggests that H.pylori decreased A20 expression through up-regulating miR-29a-3p in GES-1 and BGC823 cells infected with H.pylori. The knockdown of A20 by siRNA enhanced the migration of human gastric epithelial cells and promoted the expression of Snail, Vimentin, and N-cadherin
\end{abstract}




\section{Cellular Physiology Cell Physiol Biochem 2018;51:1250-1263

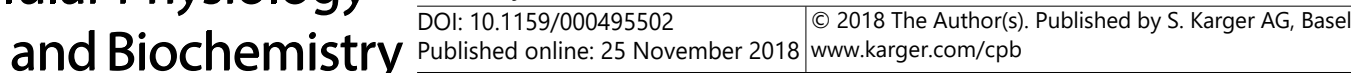 \\ Sun et al.: microRNA-29a-3p Promotes the Migration of GES-1 Cells}

and inhibited the expression of E-cadherin. Conclusion: The miR-29a-3p may act as a tumor promotive miRNA by regulating cells migration through directly targeting of A20 gene in human gastric epithelial cells infected with H.pylori.

\section{Introduction}

Over half of the population worldwide are infected with Helicobacter pylori (H.pylori), which is a gram-negative, microaerophilic, spiral-shaped, and flagellated pathogen that mainly colonizes the gastric mucosa and has coexisted with human beings for nearly sixty thousand years [1-3].Virtually all human beings infected with H.pylori may develop gastritis, a signature feature fits capacity to persist for decades, provoking a series of histological changes, ranging from the destruction of intercellular junctions to the apoptosis, hyperplasia, and even malignant transformation of epithelial cells [4, 5]. It is likely that the organism plays a causative role early in the above mentioned progression. Wong et al. reported that eradication of H.pylori significantly reduced the risk of developing gastric adenocarcinoma in individuals infected with H.pylori without premalignant lesions, providing additional evidence that H.pylori affects the early stage of gastric carcinogenesis [6]. For the past several decades, the pathogenicity of H.pylori has been widely studied, while the underlying molecular mechanisms remain to be elucidated.

miRNAs, a family of small noncoding RNAs (approximately 22 nucleotides in length), are key negative regulators of posttranscriptional gene expression, that directly bind to the $3^{\prime}$-untranslated region (3'-UTR) of target mRNAs, eventually leading to the translation degradation or inhibition of target mRNAs $[7,8]$. Aberrant expression of miRNAs is associated with several human diseases. For the past decades, a variety of miRNAs have been reported to be involved in H.pylori-associated stomach diseases by regulating the expression of target mRNAs $[9,10]$. Zhang et al. reported that H.pylori infection upregulated the expression of miR-21, which in turn downregulated the expression of reversion-inducing-cysteine-rich protein with Kazalmotifs (RECK), leading to the development of gastric cancer [11]. In addition, H.pylori infection downregulated the expression of miR-370, and then, miR-370 promoted cell proliferation by regulating the expression of FoxM1 in gastric cancer cells [12]. In our previous study, using microarray analysis, we showed that miR-29a-3p was upregulated in H.pylori-infected gastric epithelial cells (GES-1). However, this finding was not further confirmed [13]. Based on bioinformatic analysis, we further predicted that A20 might be a putative target gene of miR-29a-3p. Therefore, we develop the hypothesis that H.pylori may regulate $\mathrm{A} 20$ expression via miR-29a-3p.

The ubiquitin-editing enzyme, A20, also referred to as tumor necrosis factor $\alpha$-induced protein 3 (TNFAIP3), has been shown to negatively regulate NF- $\kappa B$ pathways by multiple mechanisms [14-17], which control numerous cellular activities including cell activation, development, and differentiation. Previous results suggested that A20, a target gene of miRlet-7f, was significantly upregulated after Mycobacterium tuberculosis (Mtb) infection, and was involved in regulating immune responses to Mtb and the control of bacterial burden [18]. In addition, the expression of A20 was robustly upregulated in murine bone marrow-derived macrophages (BMDMs) with Mycobacterium fortuitum (MF) infection [19]. However, the relevance of A20 expression and H.pylori infection in H.pylori-associated stomach diseases has not been reported so far.

Therefore, we aimed to confirm the expression profile and role of miR-29a-3p in H.pylori infection in vitro and in vivo. Exploring the regulatory mechanisms of miR-29a-3p in H.pylori infection might contribute to expanding our insights on the pathogenesis of H.pylori-related diseases. 


\section{Cellular Physiology Cell Physiol Biochem 2018;51:1250-1263 \begin{tabular}{ll|l} 
and Biochemistry Published online: 25 November 2018 & $\begin{array}{l}\text { (c) } 2018 \text { The Author(s). Published by S. Karger AG, Basel } \\
\text { www.karger.com/cpb }\end{array}$ \\
\hline
\end{tabular}}

\section{Materials and Methods}

\section{Bacterial culture}

H.pylori standard strain 26695 was kept in our laboratory (School of Medicine, Jiangsu University, Zhenjiang, Jiangsu, China), which was cultured in Columbia Agar (OXOID, UK) plates supplemented with 5\% fetal bovine serum (FBS; Wisent Inc., Quebec, Canada) under microaerophilic conditions $\left(5 \% \mathrm{O}_{2}, 10 \% \mathrm{CO}_{2}\right.$, and $85 \% \mathrm{~N}_{2}$ ) at $37^{\circ} \mathrm{C}$. The strain was harvested at the logarithmic phase and then co-cultured with gastric cells at different infection ratios and times.

\section{Cell culture}

SGC-7901, BGC-823, MGC-803, GES-1 and HEK-293T cell lines were kept in our laboratory (School of Medicine, Jiangsu University). SGC-7901, BGC-823, and HEK-293T cells were cultured in DMEM (Gibco, Grand Island, N.Y., USA) medium supplemented with 10\% FBS (Wisent Inc.). MGC-803 and GES-1 cells were maintained in RPMI-1640 (Gibco) medium containing 10\% FBS (Wisent Inc.). All cells were grown in a humidified atmosphere with $5 \% \mathrm{CO}_{2}$ at $37^{\circ} \mathrm{C}$.

\section{Patients and tissue specimens}

The gastric mucosa specimens were collected from the digestive tracts of 98 patients from December 2014 to February 2015 at the Digestive Endoscopy Center (Second People's Hospital of Changzhou, Changzhou, Jiangsu, China). Forty-six cases were H.pylori-positive. The healthy control group consisted of 52 H.pylori-negative cases. In this research, 98 clinical specimens (Supplementary Table S1) were used to analyze the expression of miR-29a-3p by qRT-PCR. In addition, forty-seven out of 98 cases were used to analyze the expression of A20 by using IHC. Twenty-four cases were H.pylori-positive, and the healthy control group included 23 H.pylori-negative cases (Supplementary Table S2).

None of the patients had received no steroidal anti-inflammatory drugs (NSAIDs), antibiotics, or proton pump inhibitor treatment at least one month before testing. H.pylori-infection status was confirmed by Rapid Urease Test (RUT) and H.pylori-specific ureC polymerase chain reaction (PCR) [20].

In this study, the patient specimens were part of the clinical specimens in the article of "Microarray analysis of Long non-coding RNA expression profiles in human gastric cells and tissues with Helicobacter pylori infection" [13].The present study was approved by the ethics committee of The Second People's Hospital of Changzhou (Supplementary Figure S1, Medical Ethics Review Report - for all supplemental material see www.karger.com/10.1159/000495502/). Written informed consents for collection and analysis of gastric mucosa were obtained from all patients.

\section{Reverse transcription and $q R T-P C R$}

Total RNA was extracted from tissues or cells using TRIzol reagent (Invitrogen, California, USA) according to the manufacturer's protocol.

For detecting the expression of miR-29a-3p, $1 \mu \mathrm{g}$ of total RNA was transcribed into cDNA using the miScript ${ }^{\circledR}$ II RT Kit (Qiagen, Valencia, CA, USA), according to the manufacturer's instructions. Then, quantitative real-time PCR (qRT-PCR) was performed with specific primers for miR-29a-3p and U6 snRNA (miScript Primer Assays, Qiagen) on ABI Step One Real-Time PCR System (Applied Bio systems). U6 snRNA was used as internal control for miRNA. All qRT-PCRs reactions were performed in triplicate and the relative expression of miRNA was calculated using $2^{-\Delta \Delta \mathrm{Ct}}$ method.

Table 1. siRNA sequences

\begin{tabular}{ll}
\hline Name & Sequence, 5 $^{\prime}$ - ${ }^{\prime}$ \\
\hline A20 siRNA-1-sense & GCGGAAAGCUGUGAAGAUATT \\
A20 siRNA-1-antisense & UAUCUUCACAGCUUUCCGCTT \\
A20 siRNA-2-sense & GCAACUGGAGUCUCUCAAATT \\
A20 siRNA-2-antisense & UUUGAGAGACUCCAGUUGCTT \\
A20 siRNA-3-sense & GGAAUGAUGAAUGGGACAATT \\
A20 siRNA-3-antisense & UUGUCCCAUUCAUCAUUCCTT \\
NC siRNA-sense & UUCUCCGAACGUGUCACGUTT \\
NC siRNA-antisense & ACGUGACACGUUCGGAGAATT \\
\hline
\end{tabular}




\section{Cellular Physiology Cell Physiol Biochem 2018;51:1250-1263 \begin{tabular}{ll|l} 
and Biochemistry Published online: 25 November 2018 & $\begin{array}{l}\text { (c) } 2018 \text { The Author(s). Published by S. Karger AG, Basel } \\
\text { www.karger.com/cpb }\end{array}$ \\
\hline
\end{tabular}}

\section{Plasmid construction and transient transfection}

Dual luciferase report vectors containing the wild type (psiCHECK-2-A20-wild-3'-UTR) and mutant type (psiCHECK-2-A20-mutant-3'-UTR) of A20 3'-UTR were constructed and confirmed by sequencing. A full-length cDNA of humanA20 was synthesized by Bioworld (Nanjing, China). Then, the product was subcloned into the pcDNA3.3(+) plasmid (Invitrogen, California, USA) to construct the recombinant plasmid, pcDNA3.0-A20.Wetransfected cells with a final concentration of 80nM of A20 siRNA (Shanghai GenePharma Co., Ltd., Shanghai, China)to silence A20 expression, with 50nM of miR-29a-3p mimic (GenePharma) to increase miR-29a-3p expression, and with $100 \mathrm{nM}$ of miR-29a-3 pinhibitor (GenePharma) to inhibit miR29a-3p function using Lipofectamine 2000(Life Technologies), according to the manufacturer's protocols. The specific sequences of human A20 siRNA and negative control siRNA are listed in Table1.

\section{Dual luciferase reporter assay}

For the dual luciferase assay, $1 \times 10^{5} \mathrm{HEK}-293 \mathrm{~T}$ cells were seeded and cultured in 24-well plates to reach approximately $70 \%$ confluence. The cells were co-transfected with $50 \mathrm{nM}$ mimics of miR-29a-3p (or 100nM inhibitors of miR-29a-3p) and 50ng of the psiCHECK-2-A20-wild-3'-UTR (or psiCHECK-2-A20-mutant-3'UTR) using Lipofectamine 2000. Twenty-four hours later, the luciferase activity of transfected cells was measured using the Dual Luciferase Reporter Assay System (Promega, Madison, WI, USA).

\section{Western blotting}

Cells were lyzed with RIPA lysis buffer (Beyotime) containing 1×Phenylmethanesulfonyl fluoride (PMSF). The protein amount of whole cell lysates was quantified by ND-1000 spectrophotometer. Proteins were separated by $10 \%$ SDS-PAGE gel and transferred into PVDF membranes. The membranes were blocked in TBST containing 5\% nonfat milk and then, incubated with the primary antibodies of A20 (Abcam), Snail (Santa Cruz), E-cadherin (Santa Cruz), Vimentein (Santa Cruz), N-cadherin (Santa Cruz), MMP-2 (Santa Cruz), MMP-9 (Santa Cruz), TIMP2 (Santa Cruz), and GAPDH (Santa Cruz) overnight at $4^{\circ} \mathrm{C}$. The membranes were washed and then, incubated with HRP-conjugated goat anti-rabbit IgG antibody (Santa Cruz) for 45 minutes at room temperature. Finally, membranes were washed and bands were visualized with ECL substrate (TransGen Biotech).

\section{Immunohistochemistry assay}

Formalin-fixed paraffin-embedded gastric mucosa tissues were deparaffinized in xylene, rehydrated through the graded alcohol of 100\%, 95\%, 90\%, 85\% and 70\%, and then, boiled for 10 minutes in citrate buffer (10mM, pH 6.0) for antigen retrieval. The endogenous peroxidase activity was suppressed by exposure to $3 \%$ hydrogen peroxide for 10 minutes. Slides were then blocked with $5 \%$ bovine serum albumin (BSA), incubated with primary antibodies against $\mathrm{A} 20$ at $4^{\circ} \mathrm{C}$ overnight, and then, incubated with secondary antibody for 45 minutes at $37^{\circ} \mathrm{C}$. Slides were visualized with 3, 3'diaminobenzidine (DAB) (BOSTER, Wuhan China), and then counterstained with hematoxylin for microscopic examination.

\section{Transwell assay}

For cell migration assay, $1 \times 10^{5}$ GES- 1 cells transfected miR-29a-3p mimics or A20 siRNA were suspended in $250 \mu \mathrm{l}$ serum-free medium and seeded in the upper chambers of CoStar Transwell chambers $(8 \mu \mathrm{m}$ pore; Corning, NY, USA), meanwhile, $600 \mu$ l culture medium containing $10 \%$ FBS was added to the lower chambers to induced cell migration. Next, the cells were incubated at $37^{\circ} \mathrm{C}$ with $5 \% \mathrm{CO}_{2}$ for 24 hours; the cells in the upper surface of the membrane were removed with a cotton swab. Cells on the bottom surface of membranes were fixed with $4 \%$ paraformaldehyde and stained with crystal violet, and then, counted under microscope with $200 \times$ magnification. Five fields per membrane were counted and the average number of cells in a field was calculated.

\section{Statistical analysis}

Quantitative data were represented as the mean \pm standard deviation (SD). The differences in the expression levels of A20 mRNA and miR-29a-3p were analyzed by Student's $t$-test using SPSS software (version20.0 SPSS Inc., Chicago, IL, USA). Statistical values of $P<0.05$ were considered significant. 


\section{Cellular Physiology Cell Physiol Biochem 2018;51:1250-1263 \begin{tabular}{ll|l} 
and Biochemistry Published online: 25 November 2018 & $\begin{array}{l}\text { (c) } 2018 \text { The Author(s). Published by S. Karger AG, Basel } \\
\text { www.karger.com/cpb }\end{array}$ \\
\hline
\end{tabular}

\section{Results}

\section{H.pylori promotes miR-29a-3p expression in vivo and in vitro}

To validate the expression of miR-29a-3p in H.pylori-positive gastric mucosa tissues and gastric epithelial-derived cell lines with H.pylori infection, we conducted qRT-PCR for 98 samples of gastric mucosa tissues and 4 gastric epithelial-derived cell lines with H.pylori infection. Our results showed that when compared with the H.pylori-negative tissues, the expression of miR-29a-3p was enhanced in H.pylori-positive tissues. The average fold change of miR-29a-3p was significantly higher in H.pylori-positive tissues than in H.pylorinegative tissues $(P=0.032$, paired Student's $t$-test; Fig. $1 \mathrm{~A})$. The expression of miR-29a-3p in four gastric epithelial-derived cell lines (SGC-7901, BGC-823, MGC-803, and GES-1) that were infected with H.pylori (standard strain 26695) was upregulated in a dose-dependent manner (Fig. 1B, 1C). In the present study, we mainly aimed to explore the role of miR-29a$3 p$ in the early stage of H. pylori infection in gastric mucosa tissues. Thus, we selected GES-1 for the next experiments.

\section{Effects of miR-29a-3p on the migration of human gastric epithelial cells}

We overexpressed miR-29a-3p by transfecting GES-1and BGC-823 cells with miR-29a$3 p$ mimics and silenced miR-29a-3p by transfecting GES- 1 and BGC-823 cells with miR29a-3p inhibitors. QRT-PCR results showed that miR-29a-3p expression was dramatically increased in GES-1 and BGC-823 cells transfected with miR-29a-3p mimics and reduced in GES-1 and BGC-823 cells transfected with miR-29a-3p inhibitors, compared with the control group transfected with negative miRNAs (Fig. 2A, 2B).

We assessed the effects of miR-29a-3p on the migration of GES-1 cells using transwell migration assay. As shown in Fig. 2C and 2D, miR-29a-3p overexpression in GES-1 cells promoted the migration of GES-1 cells. The migratory ability of GES-1 is weak since it belongs to normal gastric mucosa epithelial cells. Thus, the transwell migration assays analyzing the migration of GES-1 transfected with miR-29a-3p inhibitors were not done.

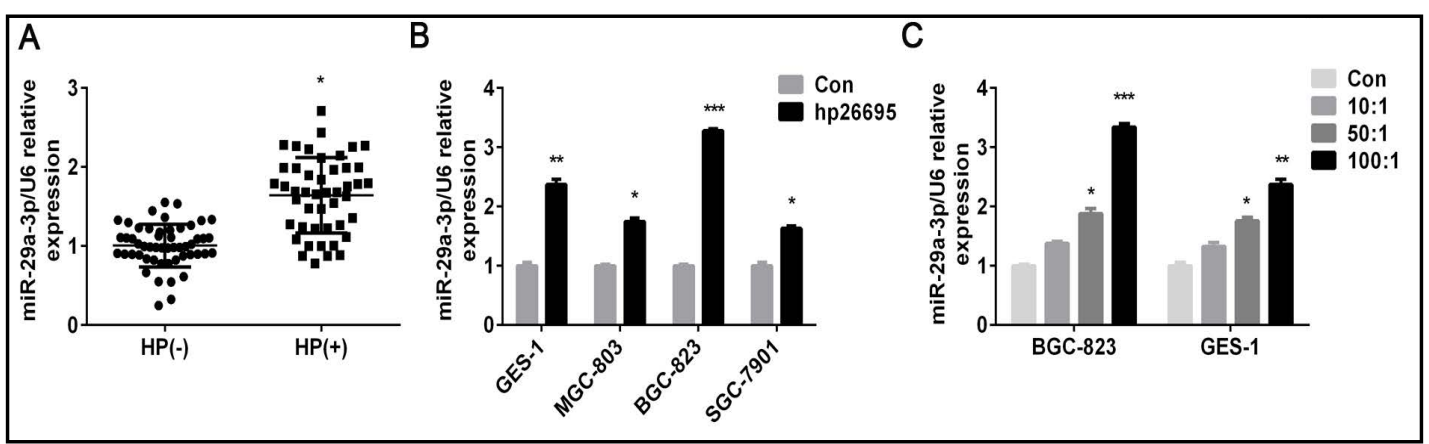

Fig. 1. Increased expression of miR-29a-3p in human gastric mucosa tissues and gastric cells with H.pylori infection. (A) qRT-PCR analysis of mRNA levels of miR-29a-3p in human gastric mucosa tissues. Each point represents one sample. "Column Hp (+)" stands for H.pylori-positive tissue samples and "column Hp (-) stands for H.pylori-negative tissue samples. (B) Co-culturing SGC-7901, BGC-823, MGC-803, and GES-1 cells with H.pylori (standard strain 26695) respectively upregulated the expression of miR-29a-3p compared with controls. Controls (the four cells without H.pylori infection) (C) BGC-823 and GES-1 cells were infected with H.pylori (standard strain 26695) at multiplicities of infection (MOIs) of 10:1, 50:1, and 100:1, respectively. The expression of miR-29a-3p was upregulated in a dose-dependent manner. Data were presented as means \pm SD from at least three separate experiments; ${ }^{*}, \mathrm{P}<0.05,{ }^{* *}, \mathrm{P}<0.01,{ }^{* * *}, \mathrm{P}<0.001$. 


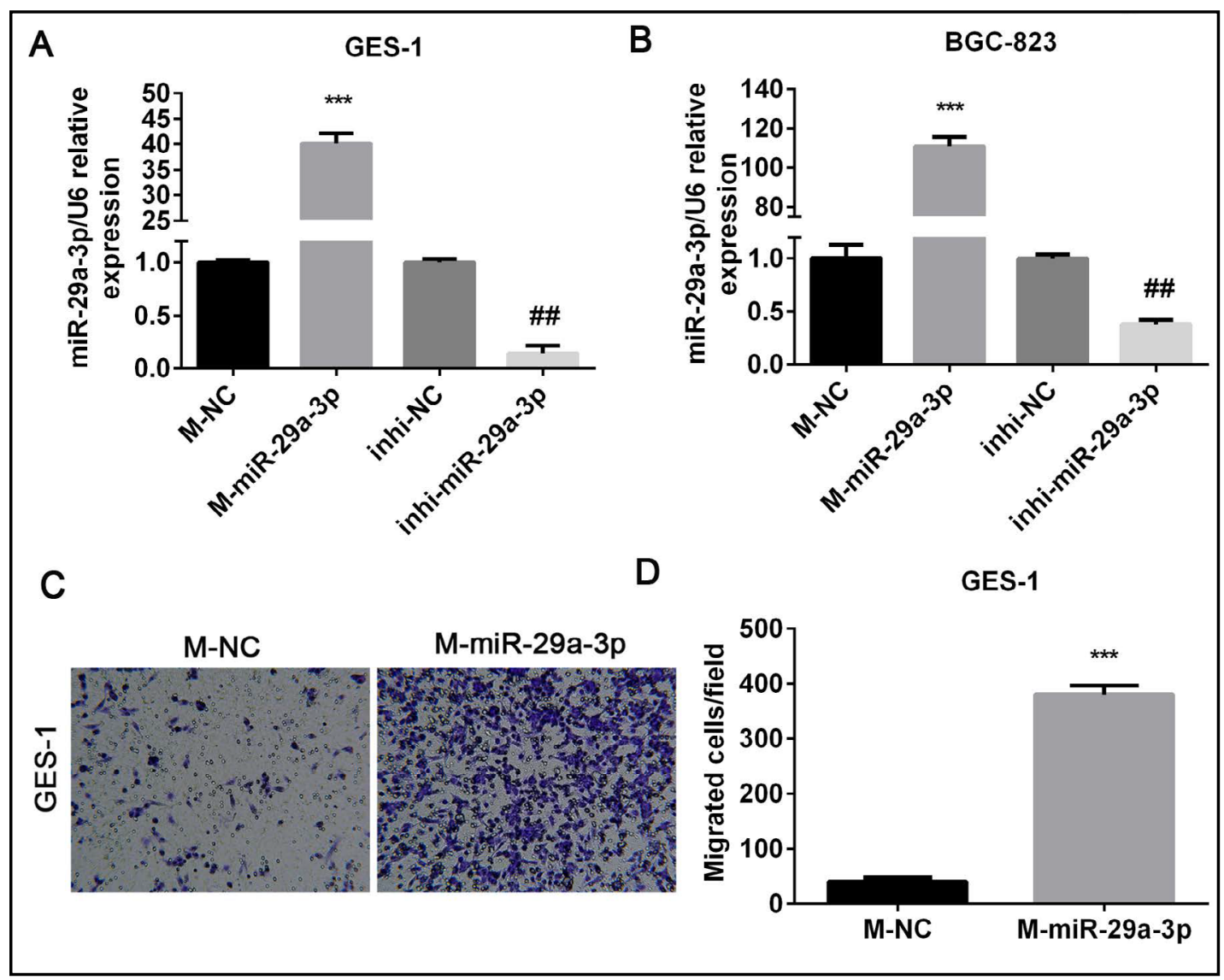

Fig. 2. Ectopic expression of miR-29a-3p in gastric epithelial cells promotes cell migration. (A and B). MiR-29a-3p expression was markedly increased in GES-1 and BGC-823 cells transfected with miR-29a-3p mimics, and decreased in GES-1 and BGC-823 cells transfected with miR-29a-3p inhibitors, compared with negative controls at 48 hours after transfection. U6 snRNA served as the endogenous control. ***, $\mathrm{P}<0.0001$ compared with M-NC, \#\#, $\mathrm{P}<0.001$ compared with inhi-NC. (C and D). The transwell assay was used to assess the effect of miR-29a-3p on the migration of GES-1 cells. Scale bar represents $100 \mu \mathrm{m}$. Magnification, 200×. Quantified results are presented as a histogram. Data are represented as the means \pm SD from 3 independent experiments. ${ }^{* * *}, \mathrm{P}<0.0001$. "M" represents mimics, "inhi" represents inhibitor, "NC" represents negative control.

A20 is negatively mediated by miR-29a-3p in human gastric cells

We used three kinds of computer-aided algorithmic programs [PicTar (http://pictar. mdc-berlin.de), TargetScan Human (http://www.targetscan.org), and miRBase (http://www. mirbase.org)] to predict A20 as a putative target gene of hsa-miR-29a-3p. As shown in Fig. $3 \mathrm{~A}, \mathrm{~A} 20$ contained a conserved binding site for miR-29a-3p in different species, suggesting that A20 was a putative target gene of miR-29a-3p. To further verify whether miR-29a-3p could directly bind to the seed sequence in the $3^{\prime}$-UTR of A20 or not, we generated wild type (WT) and mutant type (Mut) plasmids of A20 $3^{\prime}$-UTR (Fig. 3B). Using luciferase reporter assay, we found that co-transfection of plasmids of WT A20 3'-UTR and miR-29a-3p mimics reduced the luciferase activity relative to the control, whereas co-transfection of plasmids of Mut A20 3-'UTR and miR-29a-3p mimics did not affect the luciferase activity compared with the control (Fig. 3C).

Then, we performed western blotting assays to detect whether the protein levels of A20 were regulated by miR-29a-3p or not. Both BGC-823 and GES-1 cells transfected with mimics of miR-29a-3p showed that the protein levels of A20 were reduced compared with 


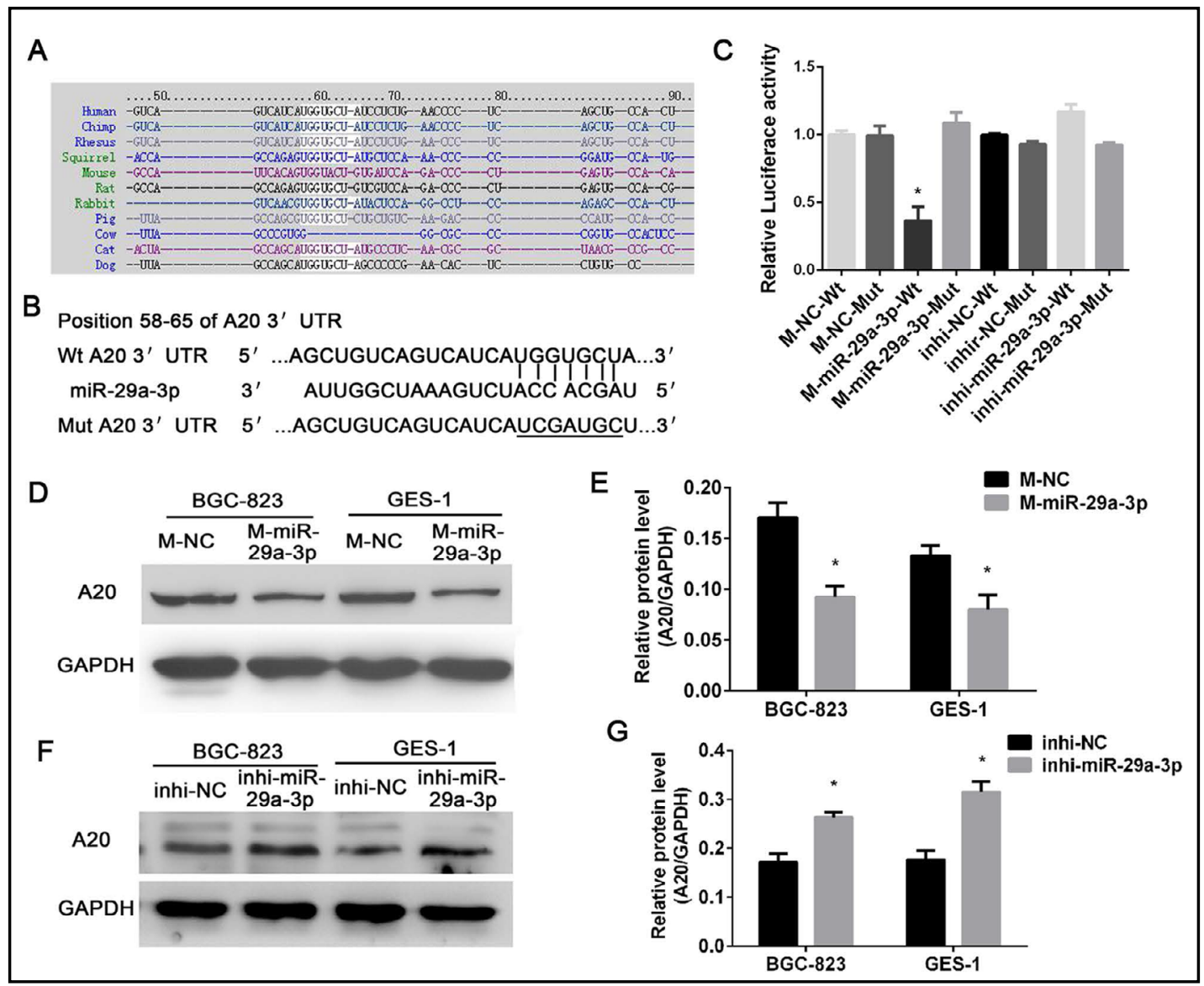

Fig. 3. miR-29a-3p directly targeted to A20 in vitro. (A) The predicted binding sites for A20 to miR-29a-3p in different species. (B)Wild type (WT) and a mutant type (Mut, indicated by black underline) of A20 3'-UTR as well as the putative seed sequences of miR-29a-3p are shown. (C)HEK-293T cells were co-transfected with luciferase reporter plasmids and mimics or inhibitors of miR-29a-3p.The luciferase reporter activity was normalized using Renilla. "Wt" stands for psiCHECK-2-A20-wild type-3'UTR reporter plasmid, "Mut" stands for psiCHECK-2-A20-mutant-3'UTR reporter plasmid; "NC" stands for negative control.*, P<0.05 versus mimic-NC+Wt. (D and E) Western blotting analysis of protein levels of A20 in BGC-823 and GES-1 cells with overexpression (mimics) of miR-29a-3p. ( $F$ and G) Western blotting analysis of protein levels of A20 in BGC-823 and GES-1 cells with inhibition (inhibitors) of miR-29a-3p. GAPDH was used as an internal reference. *, $\mathrm{P}<0.05$. "M" represents mimics; "inhi" represents inhibitor, "NC" represents negative control.

cells transfected with control miRNAs (Fig. 3D, 3E). Meanwhile, the protein levels of A20 were upregulated in BGC-823 and GES- 1 cells after inhibiting the expression of miR-29a-3p with inhibitors of miR-29a-3p (Fig. 3F, 3G). Therefore, the above findings indicate that miR29a-3p directly targets the binding seed sequence of A20 3'-UTR, and A20 is a direct target gene of miR-29a-3p.

Expression levels of A20 are downregulated in human gastric epithelial-derived cell lines and tissues with H.pylori infection

We investigated the protein expression of A20 in human gastric mucosa tissues from 47 out of 98 cases, including 23 H.pylori-negative cases and 24 H.pylori-positive cases (Supplementary Table S2) using IHC assay. We found that percentages of A20-positive cells in H.pylori-positive tissues were lower compared with in H.pylori-negative tissues $(21.7 \% \mathrm{vs}$. $79.2 \%$; $P<0.01$; Fig. 4A). Patient age or sex was not associated with A20 expression. 


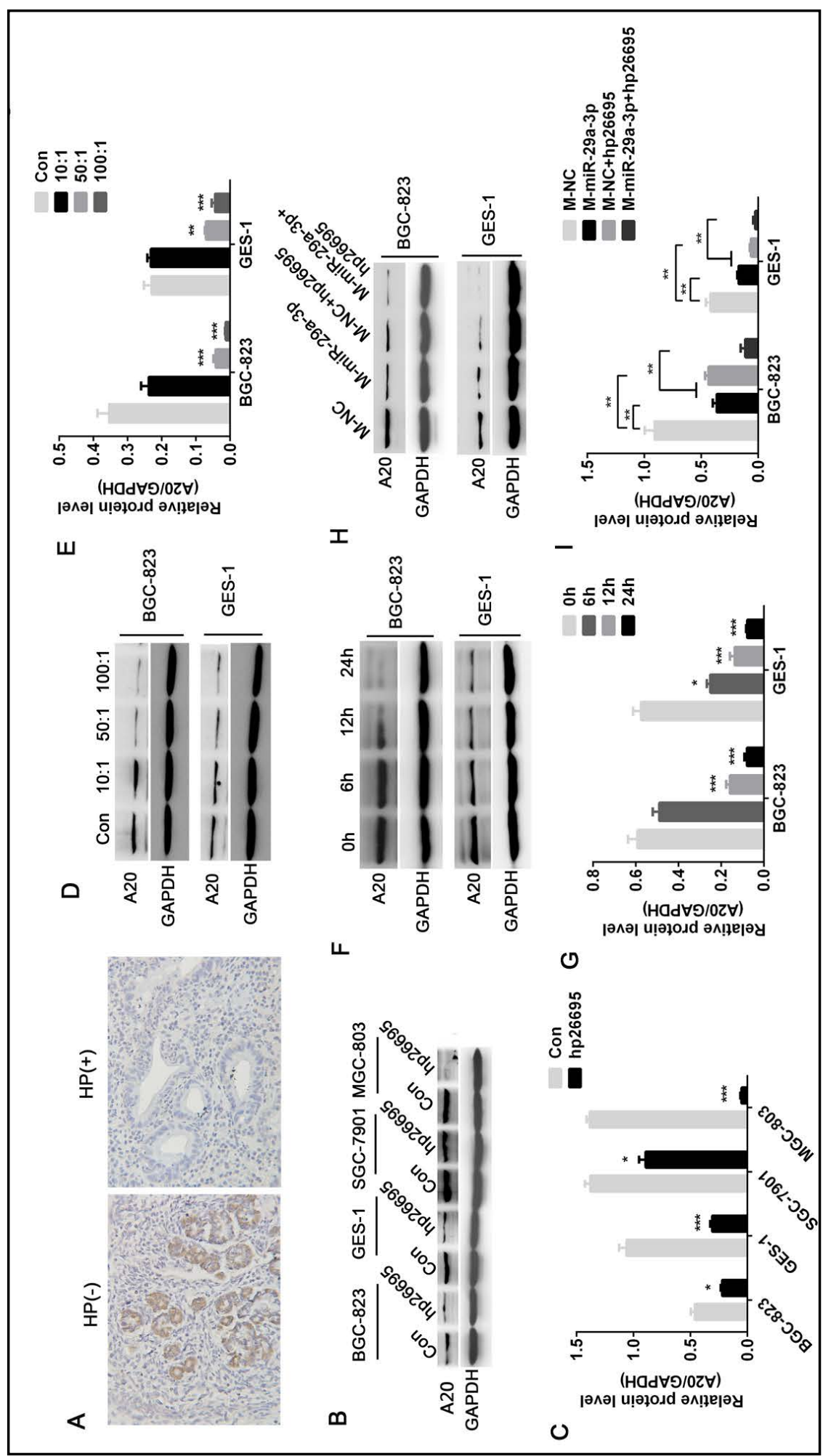

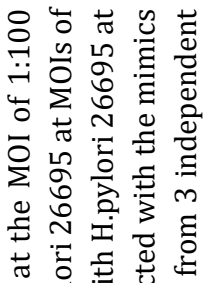

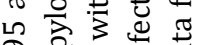

مी

N

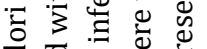

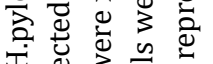

王岕氙焉

声 $\cong 00$

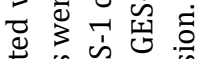

巡牙 뒁

ป

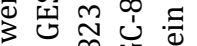

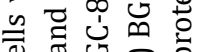

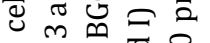

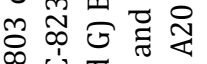

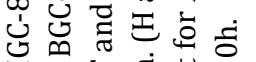

汕这 흥

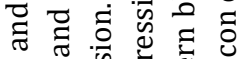

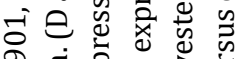

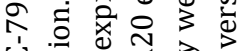

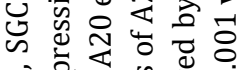

i $\begin{gathered}0 \\ 0\end{gathered}$

突

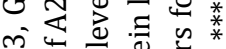

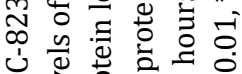

远艺市

丁.

उ

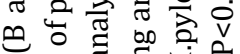

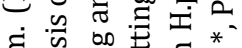

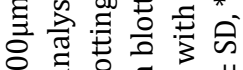

으을 $\approx+1$

bo 0 远

记

के

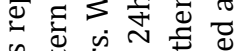

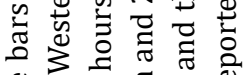

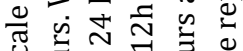

น

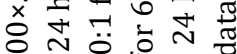

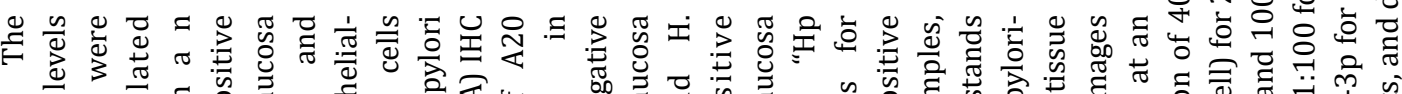

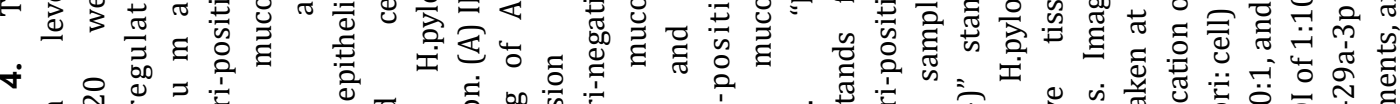

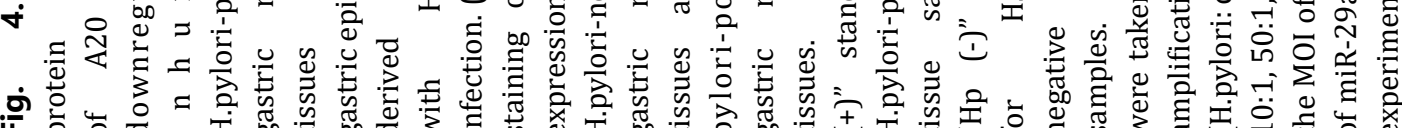

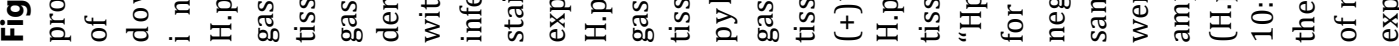




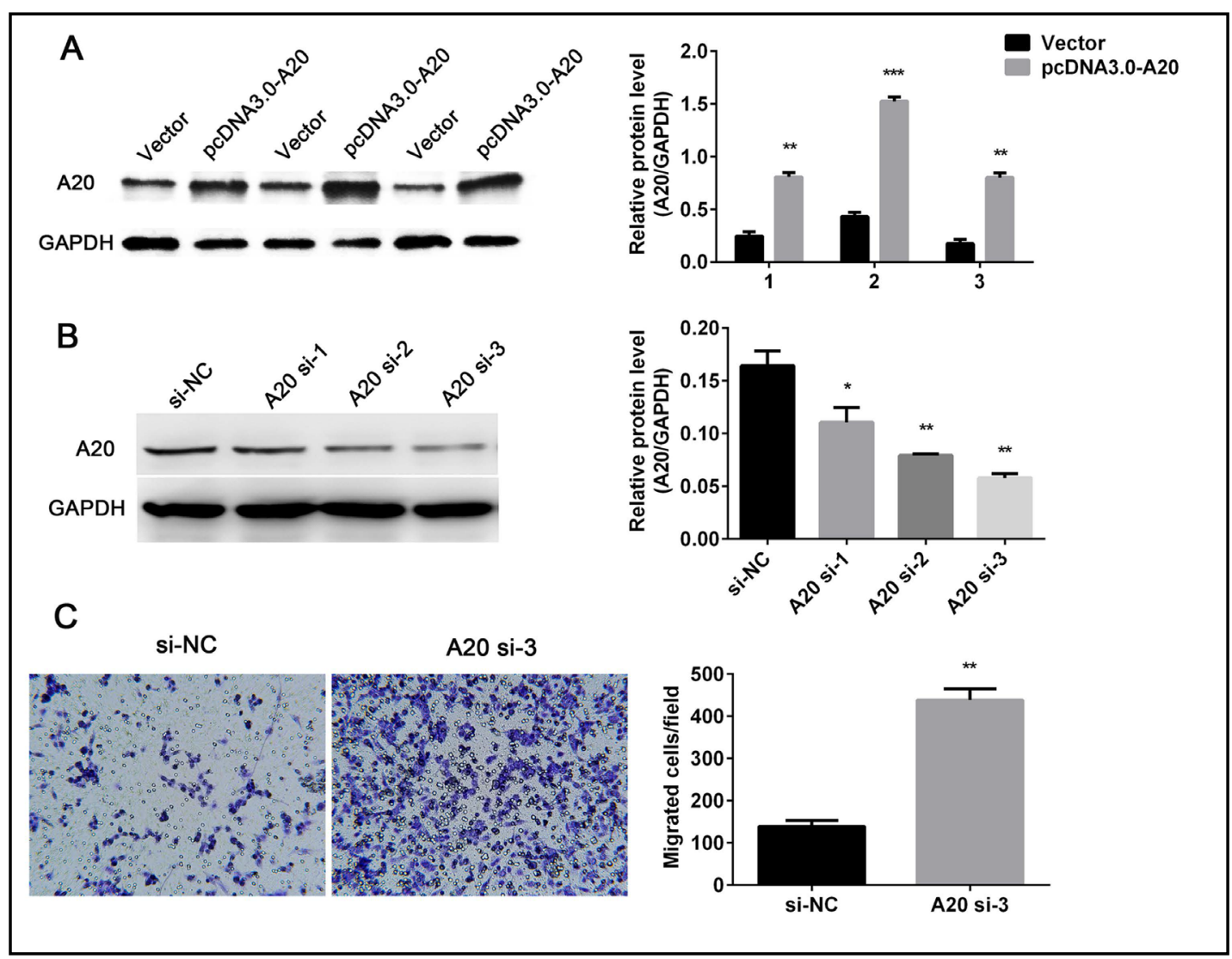

Fig. 5. A20 silencing promotes the migration of GES-1 cells. (A) pcDNA3.0-A20 vector efficiently increased the A20 expression in GES- 1 cells. ${ }^{* *}, \mathrm{P}<0.01,{ }^{* * *}, \mathrm{P}<0.001$, versus GES- 1 cells transfected with pcDNA3.0 blank vector. (B) The expression of A20 was dramatically reduced in GES-1 cells transfected with A20 siRNA. *, $\mathrm{P}<0.05$, **, $\mathrm{P}<0.01$, versus siRNA negative control. (C) The transwell assay was used to evaluate the migratory ability of GES-1 cells transfected with A20 siRNA-3. Silencing of A20 promoted the migration of GES-1 cells (200 × magnifications). ${ }^{* *}, \mathrm{P}<0.01$, versus siRNA negative control.

Next, we assessed whether or not infection with H.pylori (standard strain 26695) was associated with reduced A20 expression. Western blotting results show that the protein levels of A20 were reduced in 4 gastric epithelial-derived cell lines (SGC-7901, BGC-823, MGC-803, and GES-1) infected with H.pylori in vitro (Fig. 4B and 4C). In order to measure whether the downexpression of A20 was time- and dose- dependent or not, BGC-823 and GES-1 cells were co-cultured with H.pylori standard strain 26695 for different times and at different MOIs. Results suggested that the protein expression of A20 was gradually reduced in BGC-823 and GES-1 cells with increased time of co-culturing and with increased MOIs of H.pylori (Fig. 4D, 4E and 4F, 4G).

\section{H.pylori infection decreases A20 expression through upregulation of miR-29a-3p}

With H.pylori infection, the protein expression of A20 was decreased, and the mRNA level of miR-29a-3p was upregulated in vivo and in vitro. Meanwhile, A20 was a direct target gene of miR-29a-3p. Therefore, we developed the hypothesis that H.pylori infection decreased A20 expression through upregulation of miR-29a-3p. Subsequently, we infected BGC-823 and GES-1 cells that were transfected with mimics or inhibitors of miR-29a-3p with H.pylori (standard strain 26695). Western blotting results show that miR-29a-3p mimics significantly blocked H.pylori-induced downregulation of A20 in BGC-823 and GES-1 cells (Fig. 4H and 4I), which suggests that miR-29a-3p-mediated posttranscriptional regulation is involved in H.pylori-induced downregulation of A20 protein expression. 


\section{Cellular Physiology Cell Physiol Biochem 2018;51:1250-1263

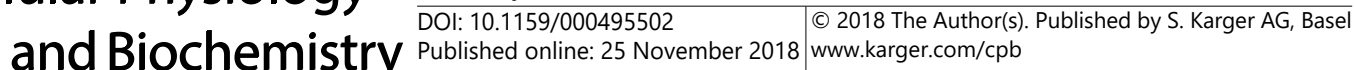

Silencing of A20 expression in gastric epithelial cells promoted migration

So far, the effects of A20 on gastric epithelial cells have not been fully illuminated. To explore the functions of A20, we transfected GES-1 cells with pcDNA3.0-A20 (overexpressing vector). A20 in GES-1 cells transfected with pcDNA3.0-A20 was overexpressed compared with GES-1 cells transfected with pcDNA3.0 (control vector) (Fig. 5A). In addition, we knocked down A20 expression using A20-specific siRNA. Just as in Fig. 5B, transfection with specific siRNA-3 against A20 (A20 si-3) into GES-1 cells, as compared with transfection with the siRNA-NC (si-NC), markedly decreased A20 expression and dramatically promoted the migration of GES-1 cells (Fig. 5C).

Next, we examined the expression of several molecular markers associated with migration in GES-1 cells using western blotting. Just as in Fig. 6C, the expression of MMP-2 and MMP-9 were markedly decreased, while the expression of TIMP2 was significantly increased in GES-1 over expression group, when compared to the blank vector group. In contrast, the silencing of A20 led to a modest increase of the MMP-9 level, while the expression of TIMP2 protein was distinctly reduced in GES-1 cells (Fig. 6D). In summary, these results suggest that A20 expression could affect the migration of gastric epithelial cell in vitro.

\section{Silencing of A20 induces EMT in gastric epithelial cells}

Prior to the migration and invasion of epithelial cells is often a developmental and morphological alternation called epithelial mesenchymal transition (EMT) [21, 22]. Next, the protein expression of a serious of indicators of EMT was measured. As shown in Fig. $6 \mathrm{~A}$, the expression of mesenchymal markers, Snail, Vimentin, and N-cadherin, were reduced in A20-overexpressed cells, while the expression of the epithelial marker, E-cadherin, was dramatically increased. In contrast, the silencing of A20 led to a modest decrease of E-cadherin in GES-1 cells, however, the protein expression of Snail, Vimentin, and N-cadherin were increased as expected (Fig. 6B). Overall, these results indicate that silencing of A20 involved in the process of EMT and intensive the migratory ability of gastric epithelial cells in vitro.

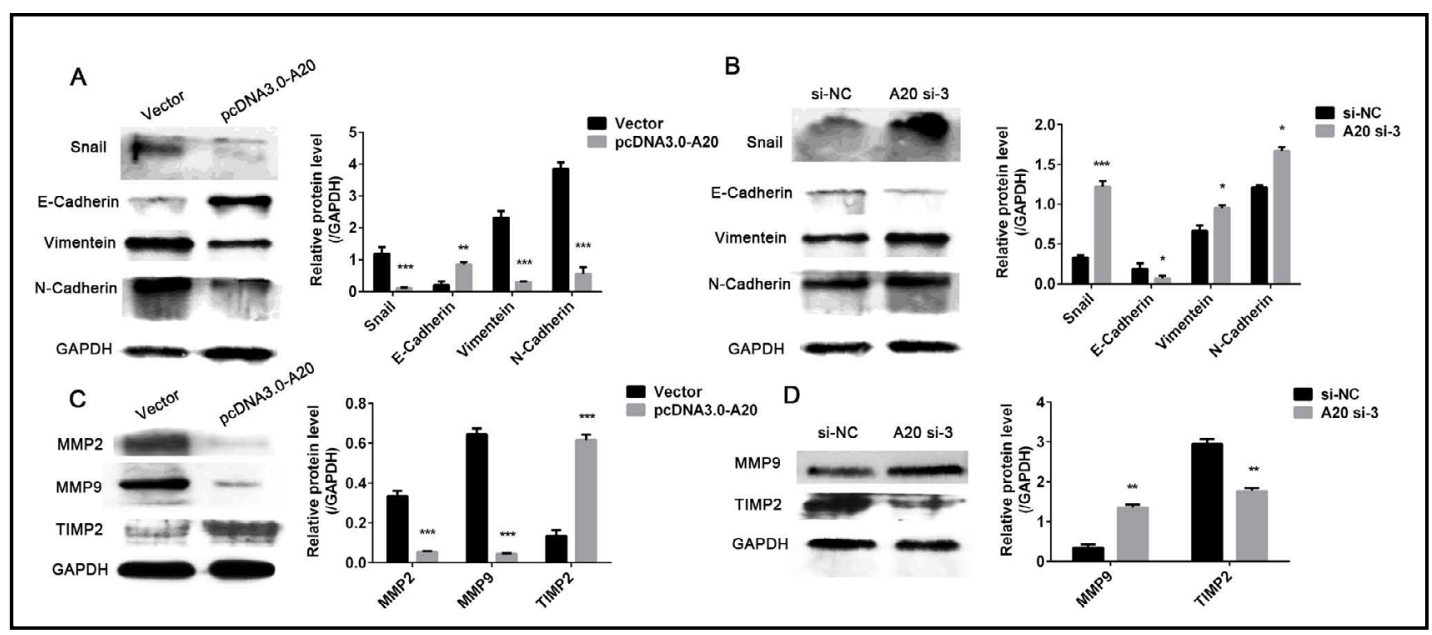

Fig. 6. A20 affects the expression of epithelial-mesenchymal transition (EMT) pathway-associated markers. (A) High A20 could increase the expression of E-cadherinand reduced the expression of Snail, Vimentein, and N-cadherin in GES-1 cells. (B) Silencing of A20 affected the EMT phenotype through decreasing the protein expression of E-caherin and increased the protein expression of Snail, Vimentin, and N-cadherin expression in GES-1 cells. (C and D) Expression of the migration-related gene was detected by western blotting in GES-1 cells at 48 hours after transfection with pcDNA3.0-A20 or A20 si-3. 


\section{Cellular Physiology Cell Physiol Biochem 2018;51:1250-1263 and Biochemistry Published \begin{tabular}{l|l} 
DOI: 10.1159/000495502 & $\begin{array}{l}\text { @ } 2018 \text { The Author(s). Published by S. Karger AG, Basel } \\
\text { www.karger.com/cpb }\end{array}$
\end{tabular} \\ Sun et al.: microRNA-29a-3p Promotes the Migration of GES-1 Cells}

\section{Discussion}

Following adherence and colonization of the gastric mucosa, H.pylori could induce chronic gastric inflammation, which is regarded as the strongest risk factor for the development of atrophic gastritis, intestinal metaplasia, dysplasia, and ultimately gastric adenocarcinoma $[23,24]$. H.pylori expresses several virulence factors capable of regulating the host immune system and inducing pro-inflammatory immune responses. These factors mainly include Cag (cytotoxin_associated gene) pathogenicity island and vacuolating cytotoxin A (VacA). For the past several decades, more and more researches have been focusing on revealing the molecular mechanisms of pathogenesis of Hpylori, especially at the level of regulation of gene transcription. Recently, a series of studies on miRNAs have provided our new insights on pathogenic mechanisms of H.pylori.

miR-29a, miR-29b, and miR-29c are three family members of miR-29. They have the same seed region, AGCACCA, and similar biological functions. The abnormal expression of miR-29 family is closely linked to tumorigenesis, as it is widely involved in differentiation [25], proliferation [26], apoptosis [27], and metastasis [28] of tumor cells. A previous study demonstrated that miR-29a-3p was significantly down-regulated in gastric tumor tissues compared with the adjacent gastric mucosa tissues, and the aberrant expression of miR$29 a-3 p$ affected the proliferation and metastasis of gastric tumor cells in vitro [29].In the present study, we found that the expression of miR-29a-3p was increased in H.pylori-positive gastric mucosa tissues and gastric epithelial-derived cells infected with H.pylori (Fig. 1A, 1B). In addition, miR-29a-3p could promote the migration of gastric epithelial cells (Fig. 2C). Interestingly, this was the opposite in gastric epithelial cells, compared with the gastric cancer cells. A previous study reported that miR-29a inhibited cell migration and invasion via targeting Roundabout homolog 1 in gastric cancer cells [30]. MiR-29a may be a doubleedged sword in the development of gastric cancer. It could promote the tumorigenesis of H.pylori-associated gastric cancer in the early stage, and on the other hand, inhibit the progression of gastric cancer in the late stage.

Recent data have indicated that miR-29c could enhance A20 expression, negatively regulating IAV-induced NF- $\mathrm{KB}$ activation in A549 cells and miR-29c-mediated regulation for A20 was independent of the seed sequence of A20 3'-UTR [31]. Moreover, Mumtaz et al. described that miR-29b acted as a decoy for the RNA binding protein, HuR, to protect A20 transcripts from HuR-mediated degradation in sarcoma tumor cells [32]. In the present study, we also showed that miR-29a-3p was associated with A20 expression. We transfected gastric epithelial-derived cells with miR-29a-3p mimics and miR-29a-3p inhibitors and found that expression levels of A20 were significantly reduced and increased, respectively (Fig. 3D, 3F). In addition, our data, in combination with the dual luciferase reporter assay (Fig. 3C), suggested that miR-29a-3p directly targeted the binding site located at A20 3'-UTR, and that A20 was a direct target of miR-29a-3p.

Furthermore, we demonstrated that A20 was expressed at a significantly lower level in H.pylori-positive gastric mucosal tissues than in H.pylori-negative gastric mucosal tissues (Fig. 4A). Similarly, protein levels of A20 in H.pylori-infected gastric epithelial-derived cells were decreased (Fig. 4B). Next, we further analysis of the relationship between the expression of A20, miR-29a-3p and H.pylori. The results showed that H.pylori infection decreased A20 expression by upregulating miR-29a-3p (Fig. 4H). In addition, we also confirmed that the silencing of A20 affected the expression of MMP2, MMP-9, and TIMP2 (Fig. 6C and 6D), resulting in gastric epithelial cells migration (Fig. 5C).

Epithelial-mesenchymal transition (EMT) is a process that includes molecular changes, decreased cell-cell junction and adhesion, and increased cell motility and causes cells to gain migratory and invasive properties, key events in cancer metastasis [33-36]. EMT can be determined by the increase of mesenchymal markers (Vimentin, Snail and N-cadherin) along with the decrease of epithelial markers (E-cadherin) [37]. Our data suggested that the expression of the E-cadherin was reduced, while the expression of Snail, Vimentein, and N-cadherin were enhanced in A20-downregulated GES-1 cells (Fig. 6B). In contrast, 


\section{Cellular Physiology Cell Physiol Biochem 2018;51:1250-1263

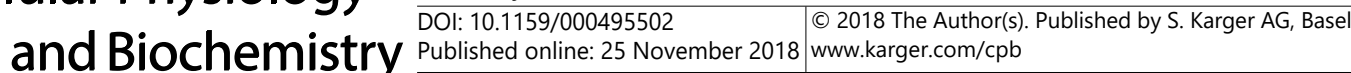 \\ Sun et al.: microRNA-29a-3p Promotes the Migration of GES-1 Cells}

the expression of E-cadherin was increased, while the expression of Snali, Vimentein, and $\mathrm{N}$-cadherin were decreased in A20-overexpressed GES-1 cells (Fig. 6A). Therefore, these results indicate that the silencing of A20 may induce the EMT to promote the development of gastric cancer.

\section{Conclusion}

Our study demonstrates that miR-29a-3p was upregulated in H.pylori-positive gastric mucosal tissues and gastric epithelial-derived cells with H.pylori infection. In addition, H.pylori infection reduced A20 protein expression via upregulating miR-29a-3p. Both overexpression of miR-29a-3p and silencing of A20 promote the migration of gastric epithelial cells migration, which was associated with changes of EMT markers. In this study, our results reveal a new pathogenic mechanism of H.pylori infection, and indicate that miR29 a-3p may be a rational therapeutic target for the treatment of H.pylori-associated stomach diseases in the future.

\section{Abbreviations}

H.pylori (Helicobacter pylori); miRNA (microRNA); IHC (Immunohistochemistry); 3'UTR ( $3^{\prime}$-untranslated region); siRNA (Small interfering RNA); qRT-PCR (Quantitative realtime polymerase chain reaction).

\section{Acknowledgements}

This work was supported by the National Natural Science Foundation of China (Grant numbers 81772157 and 81271795), the Specialized Research Fund for the Doctoral Program of Higher Education of China (Grant number 20123227110008), the China Postdoctoral Science Foundation (Grant number 2016M591790), the Natural Science Foundation of Jiangsu Province, China (Grant number BK20161345) and the Key Science Research Project in Colleges and Universities of Jiangsu Province, China (Grant number 17KJA320001).

\section{Disclosure Statement}

The authors declare no conflicts of interest.

\section{References}

1 Moss SF, Sood S: Helicobacter pylori. Curr Opin Infect Dis 2003;16:445-451.

-2 Linz B, Balloux F, Moodley Y, Manica A, Liu H, Roumagnac P, Falush D, Stamer C, Prugnolle F, van der Merwe SW, Yamaoka Y, Graham DY, Perez-Trallero E, Wadstrom T, Suerbaum S, Achtman M: An African origin for the intimate association between humans and Helicobacter pylori. Nature 2007;445:915-918.

-3 Peek RM Jr, Crabtree JE: Helicobacter infection and gastric neoplasia. J Pathol 2006;208:233-248.

4 Xia HH, Talley NJ: Apoptosis in gastric epithelium induced by Helicobacter pylori infection: implications in gastric carcinogenesis. Am J Gastroenterol 2001;96:16-26.

5 Watari J, Chen N, Amenta PS, Fukui H, Oshima T, Tomita T, Miwa H, Lim KJ, Das KM: Helicobacter pylori associated chronic gastritis, clinical syndromes, precancerouslesions, and pathogenesis of gastric cancer development. World J Gastroenterol 2014;20:5461-5473. 


\section{Cellular Physiology Cell Physiol Biochem 2018;51:1250-1263 and Biochemistry \begin{tabular}{l|l} 
DOI: $10.1159 / 000495502$ & 2018 The Author(s). Published by S. Karger AG, Basel
\end{tabular}

6 Wong BC, Lam SK, Wong WM, Chen JS, Zheng TT, Feng RE, Lai KC, Hu WH, Yuen ST, Leung SY, Fong DY, Ho J, Ching CK, Chen JS: Helicobacter pylori eradication to prevent gastric cancer in a high-risk region of China: a randomized controlled trial. JAMA 2004;291:187-194.

7 He L, Hannon GJ: MicroRNAs: small RNAs with a big role in gene regulation. Nat Rev Genet 2004;5:522-531.

8 Chen K, Rajewsky N: The evolution of gene regulation by transcription factors and microRNAs. Nat Rev Genet 2007;8:93-103.

-9 Matsushima K, Isomoto H, Inoue N, Nakayama T, Hayashi T, Nakayama M, Nakao K, Hirayama T, Kohno S: MicroRNA signatures in Helicobacter pylori-infected gastric mucosa. Int J Cancer 2011;128:361-370.

10 Cadamuro AC, Rossi AF, Maniezzo NM, Silva AE: Helicobacter pylori infection: host immune response, implications on gene expression and microRNAs. World J Gastroenterol 2014;20:1424-1437.

-11 Zhang Z, Li Z, Gao C, Chen P, Chen J, Liu W, Xiao S, Lu H: miR-21 plays a pivotal role in gastric cancer pathogenesis and progression. Lab invest 2008;88:1358-1366.

12 Feng Y, Wang L, Zeng J, Shen L, Liang X, Yu H, Liu S, Liu Z, Sun Y, Li W, Chen C, Jia J: FoxM1 is overexpressed in Helicobacter pylori-induced gastric carcinogenesis and is negatively regulated by miR-370. Mol Cancer Res 2013;11:834-844.

13 Hong Z, Qiang W, Yao Y, Jian F, Sun F, Ying N, Shen Y, Wang H, Shao S: Microarray analysis of Long non-coding RNA expression profiles in human gastric cells and tissues with Helicobacter pylori Infection. BMC Med Genomics 2015;8:84.

14 Shembade N, Harhaj E: A20 inhibition of NFкB and inflammation: Targeting E2:E3 ubiquitin enzyme ecomplexes. Cell Cycle 2010;9:2481-2482.

15 Wertz IE, O’Rourke KM, Zhou H, Eby M, Aravind L, Seshagiri S, Wu P, Wiesmann C, Baker R, Boone DL, Ma A, Koonin EV, Dixit VM: De-ubiquiti nation and ubiquitin ligase domains of A20 downregulate NF-B signalling. Nature 2004;430:694-699.

-16 Coornaert B, Carpentier I, Beyaert R: A20: Central Gatekeeper in Inflammation and Immunity. J Biol Chem 2009;284:8217-8221.

17 Catrysse L, Vereecke L, Beyaert R, van Loo G: A20 in inflammation and autoimmunity. Trends Immunol 2014;35:22-31.

-18 Kumar M, Sahu SK, Kumar R, Subuddhi A, Maji RK, Jana K, Gupta P, Raffetseder J, Lerm M, Ghosh Z, van Loo G, Beyaert R, Gupta UD, Kundu M, Basu J: MicroRNA let-7 Modulates the Immune Response to Mycobacterium tuberculosis Infection via Control of A20, an Inhibitor of the NF- $\kappa B$ Pathway. Cell Host Microbe 2015;17:345-356.

19 Lee GJ, Lee HM, Kim TS, Kim JK, Sohn KM, Jo EK: Mycobacterium fortuitum induces A20 expression that impairs macrophage inflammatory responses. Pathog Dis 2016;74:pii:ftw015.

-20 Smith SI, Oyedeji KS, Arigbabu AO, Cantet F, Megraud F, Ojo OO, Uwaifo AO, Otegbayo JA, Ola SO, Coker AO: Comparison of three PCR methods for detection of Helicobacter pylori DNA and detection of cagA gene in gastric biopsyspecimens. World J Gastroenterol 2004;10:1958-1960.

21 Gavert N, Ben-Ze'ev A: Epithelial-mesenchymal transition and the invasive potential of tumors. Trends Mol Med 2008;14:199-209.

-22 Voulgari A, Pintzas A: Epithelial-mesenchymal transition in cancer metastasis: Mechanisms, markers and strategies to overcome drug resistance in the clinic. Biochim Biophys Acta 2009;1796:75-90.

23 Peek RM Jr, Blaser MJ: Helicobacter pylori and gastrointestinal tract adenocarcinomas. Nat Rev Cancer 2002;2:28-37.

-24 Kamada T, Kusunoki H, Hata J, Haruma K: Helicobacter pylori infection and development of gastric cancer. Nihon Shokakibyo Gakkai Zasshi 2005;102:673-680.

25 Kapinas K, Kessler CB, Delany AM: MiR-29 suppression of osteonectin in osteoblasts: Regulation during differentiation and by canonical Wnt signaling. J Cell Biochem 2010;108:216-224.

-26 Li Y, Wang F, Xu J, Ye F, Shen Y, Zhou J, Lu W, Wan X, Ma D, Xie X: Progressive miRNA expression profiles in cervical carcinogenesis and identification of HPV-related target genes for miR-29. J Pathol 2011;224:484495.

27 Pekarsky Y, Koval A, Hallas C, Bichi R, Tresini M, Malstrom S, Russo G, Tsichlis P, Croce CM: Tcl1 enhances Akt kinase activity and media tesits nuclear translocation. Proc Natl Acad Sci U S A 2000;97:3028-3033.

-28 Gebeshuber CA, Zatloukal K, Martinez J: miR-29a suppresses tristetraprolin, which is a regulator of epithelial polarity and metastasis. EMBO Rep 2009;10:400-405. 


\section{Cellular Physiology Cell Physiol Biochem 2018;51:1250-1263

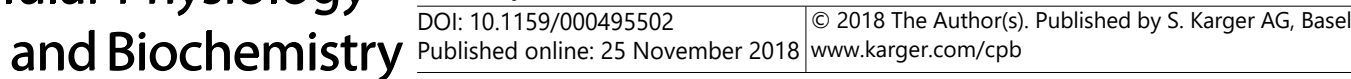 \\ Sun et al.: microRNA-29a-3p Promotes the Migration of GES-1 Cells}

29 Zhao Z, Wang L, Song W, Cui H, Chen G, Qiao F, Hu J, Zhou R, Fan H: Reduced miR-29a-3p expression is linked to the cell proliferation and cell migration in gastric cancer. World J Surg Oncol 2015;13:101.

-30 Liu X, Cai J, Sun Y, Gong R, Sun D, Zhong X, Jiang S, He X, Bao E, Yang L, Li Y: MicroRNA-29a inhibits cell migration and invasion via targeting Roundabout homolog 1 in gastric cancer cells. Mol Med Rep 2015;12:3944-3950.

31 Zhang X, Dong C, Sun X, Li Z, Zhang M, Guan Z, Duan M: Induction of the cellular miR-29c by influenza virus inhibits the innate immune response through protection of A20 mRNA. Biochem Biophys Res Commun 2014;450:755-761.

-32 Balkhi MY, Iwenofu OH, Bakkar N, Ladner KJ, Chandler DS, Houghton PJ, London CA, Kraybill W, Perrotti D, Croce CM, Keller C, Guttridge DC: miR-29 acts as a decoy in sarcomas to protect the tumor suppressor A20 mRNA from degradation by HuR. Sci Signal 2013;6:ra63.

-33 Liu H, Zhen Q, Fan Y: LncRNA GHET1 promotes esophageal squamous cellcarcinoma cells proliferation and invasion via induction of EMT. Int J Biol Markers 2017;32:e403-e408.

-34 Pan JX, Qu F, Wang FF, Xu J, Mu LS,Ye LY, Li JJ: Aberrant SERPINE1 DNA methylation is involved in carboplatin induced epithelial-mesenchymal transition in epithelial ovarian cancer. Arch Gynecol Obstet 2017;296:1145-1152.

-35 Liu W, Zhang B, Xu N, Wang MJ, Liu Q: miR-326 regulates EMT and metastasis of endometrial cancer through targeting TWIST1. Eur Rev Med Pharmacol Sci 2017;21:3787-3793.

-36 Lv ZD, Wang HB, Liu XP, Jin LY, Shen RW, Wang XG, Kong B, Qu HL, Li FN, Yang QF: Silencing of Prrx2 Inhibits the Invasion and Metastasis of Breast Cancer both In vitro and In vivo by Reversing Epithelial-Mesenchymal Transition. Cell Physiol Biochem 2017;42:1847-1856.

37 Nieto MA, Huang RY, Jackson RA, Thiery JP: EMT: 2016. Cell 2016;166:21-45. 